\title{
A multicenter, randomized, double-blind, placebo-controlled trial evaluating the efficacy and safety of Tong Luo Hua Shi capsule, a modernized Tibetan medicine, in patients with rheumatoid arthritis
}

Wei Liu ${ }^{1 * \dagger}$, Yuan-Hao Wu ${ }^{1 \dagger}$, Si-Yuan Hu' ${ }^{1}$, Cheng-Liang Zhong ${ }^{1}$, Ming-Li Gao ${ }^{2}$, Dong-Wu Liư ${ }^{2}$, Hai-Yun Wang ${ }^{3}$, Mu-Zhi Chen ${ }^{3}$, Yue-Jin Song ${ }^{4}$, Ben-Zha-Xi Yang ${ }^{5}$, Qing-Shan Zheng ${ }^{6}$, Hua Yao ${ }^{7}$, Xue-Bing Qi ${ }^{8}$ and Gang Li ${ }^{8}$

\begin{abstract}
Background: Tong Luo Hua Shi (TLHS) is a new formulation of the traditional Tibetan medicine Wu-wei-gan-lu that has been used for the treatment of rheumatoid arthritis (RA) for hundreds of years in China. This study aimed to evaluate the efficacy and safety of TLHS in patients with RA.

Methods: This was a randomized, double-blind, placebo-controlled, dose-finding study performed in patients with active RA from five medical centers. Patients received three doses (4.8, 3.6, or $2.4 \mathrm{~g} /$ day po) of TLHS or placebo (tid po) for 8 weeks. Blood sampling, physical examination, and assessment of the American College of Rheumatology (ACR) $20 \%$ improvement (ACR20) criteria were performed before and every 2 weeks after starting treatment. The primary endpoint was the ACR20. The secondary endpoints included safety.

Results: A total of 240 participants were screened and 236 patients were randomized ( $n=59 /$ group); 20 dropped out. After 8 weeks, ACR2O improvements in the TLHS $4.8 \mathrm{~g}$ and $3.6 \mathrm{~g}$ groups were significantly higher than in the placebo group $(P<0.01$ and $P<0.05$, respectively). ACR50 improvement in the TLHS $4.8 \mathrm{~g}$ group was significantly higher compared with the placebo group $(P<0.01)$. Symptoms of RA were significantly relieved in the TLHS groups. In the TLHS groups, insomnia $(n=1)$, gastroenteric reactions $(n=2)$, arrhythmia $(n=1)$, and minor hepatic lesion $(n=1)$ were reported; in the placebo group, hepatic dysfunction $(n=1)$ was reported $(P=0.878)$.
\end{abstract}

Conclusions: TLHS improved the symptoms of patients with RA according to the ACR20. Moreover, TLHS was safe. Trial registration: Chinese Clinical Trial Registry: ChiCTR-TRC-12003871. Registered on 1 January 2012.

Keywords: Rheumatoid arthritis, Tibetan medicine, Antirheumatic therapy, Tong Luo Hua Shi capsules, Wu-weigan-lu decoction

\footnotetext{
* Correspondence: fengshiliuwei@163.com

${ }^{\dagger}$ Equal contributors

${ }^{1}$ First Teaching Hospital of Tianjin University of Traditional Chinese Medicine, An-shan-xi Road \#314, Nankai District, Tianjin 300193, China

Full list of author information is available at the end of the article
}

\section{) Biomed Central}

(c) 2016 Liu et al. Open Access This article is distributed under the terms of the Creative Commons Attribution 4.0 International License (http://creativecommons.org/licenses/by/4.0/), which permits unrestricted use, distribution, and reproduction in any medium, provided you give appropriate credit to the original author(s) and the source, provide a link to the Creative Commons license, and indicate if changes were made. The Creative Commons Public Domain Dedication waiver (http://creativecommons.org/publicdomain/zero/1.0/) applies to the data made available in this article, unless otherwise stated. 


\section{Background}

Rheumatoid arthritis (RA) is a chronic inflammatory disease characterized by persistent synovitis and progressive destruction of cartilage and bone with the presence of rheumatoid factors. RA is also associated with systemic inflammatory manifestations in addition to local inflammation of multiple joints [1-3]. The prevalence of RA is $0.35 \%$ in women and $0.13 \%$ in men [4]. Risk factors include smoking, genetic factors, elevated rheumatoid factor levels, elevated soluble tumor necrosis factor receptor II levels, coffee consumption, absence of hormonal replacement therapy, and posttraumatic stress disorder [5-9]. Treatment of RA involves the control of inflammation using anti-inflammatory drugs [1, 2].

Tong Luo Hua Shi (TLHS) capsules are a new formulation of the traditional Wu-wei-gan-lu decoction, which is one of the basic medicines in traditional Tibetan medicine for the treatments of different diseases, especially RA, and has been used for hundreds of years in China [10-12]. TLHS is made from herbs including Salvia miltiorrhiza, Ephedra intermedia, Sabina przewalskii, Myricaria paniculata, Artemisia sieversiana, Astragalus membranaceus, and Rhododendron anthopogonoides [10-12]. Previous studies of the Wu-wei-gan-lu decoction revealed its efficacy for the treatment of acute gouty arthritis [13] and RA [14, 15].

Therefore, the aim of the present study was to evaluate the efficacy and safety of TLHS in patients with RA.

\section{Methods}

\section{Study design}

This was a multicenter, randomized, double-blind, placebo-controlled, dose-finding trial. It was reported according to the recommendation of the Consolidated Standards of Reporting Trials (CONSORT) [16]. The trial was approved by the State Food and Drug Administration of China (No. 2006 L03370) and the ethical committee of The First Teaching Hospital of Tianjin University of Traditional Chinese Medicine (No. TYLL2009015). Written informed consent was obtained from each participant before enrollment. The trial was registered with the Chinese Clinical Trial Registry (registration number: ChiCTRTRC-12003871; date of registration: 1 January 2012). The manuscript was in accordance with the populated CONSORT checklist (see Additional file 1) and flow diagram (see Additional file 2).

This study took place in five centers: the First Teaching Hospital of Tianjin University of Traditional Chinese Medicine, the Affiliated Hospital of Liaoning University of Traditional Chinese Medicine, the Traditional Chinese Medicine Hospital of Xinjiang Uygur Autonomous Region, the Hubei Hospital of Traditional Chinese Medicine, and the Tibetan Medicine Hospital of Qinghai Province.

\section{Participants}

Participants $(n=236)$ were recruited between May 2009 and June 2011. Participants had to have been diagnosed with RA according to the American College of Rheumatology (ACR) 1991 revised criteria [17]. All participants had to have an ACR functional class of I, II, or III, and be in radiographic stage I, II, or III.

Exclusion criteria were: (1) pregnant or lactating women, and women of child-bearing age who were not using an effective method of contraception; (2) severe disability; (3) history of serious allergic reactions; (4) any other concurrent rheumatic disease such as systemic lupus erythematosus, Sjogren's syndrome, or severe osteoarthritis; (5) significant cardiac, hematologic, respiratory, neurological, endocrine, renal, hepatic, gastrointestinal, or psychotic disease; (6) active recurrent infection; (7) alcoholism or drug dependency; or (8) psychological disorder.

Nonsteroidal anti-inflammatory drugs (NSAIDs) were discontinued at least 30 days before participation. Participants receiving a stable dosing regimen of the same glucocorticoids, e.g., prednisolone (10 mg daily maximum), or disease-modifying antirheumatic drugs (DMARDs) prior to entering the study were allowed.

If severe pain or complications occurred in a patient, making it impossible to participate in the study, as confirmed by the investigator, the patient was removed from the study. Poor compliance to treatment or study protocol led to removal from the study. Otherwise, patients could drop out of the study if they wished to; the reasons for dropping out were recorded. If a patient dropped out because of poor treatment effect, this patient was analyzed as achieving no treatment efficacy.

\section{Study medication and administration}

The TLHS and placebo capsules were provided by Lanzhou Heshengtang Pharmaceutical Co., Ltd. (Lanzhou, China). Placebos were supplied in the form of capsules matched for weight, shape, and color.

The patients were randomized to receive $4.8,3.6$, or $2.4 \mathrm{~g} /$ day (three pills/day) of TLHS po or placebo po. All patients consumed four capsules each time and the dose was adjusted using a combination of TLHS and placebo capsules. An independent statistician prepared sequential sealed envelopes based on a random number table generated using SAS 9.2 (SAS Institute, Cary, NC, USA). Randomization was implemented without blocks. The envelopes and the allocation sequence were managed by a statistician. When a patient was recruited, the treating physician phoned the independent statistician, who then opened the next envelope and phoned the pharmacist to state the allocation. Capsules were prepared by the pharmacist. Patients and physicians were blinded to grouping. 


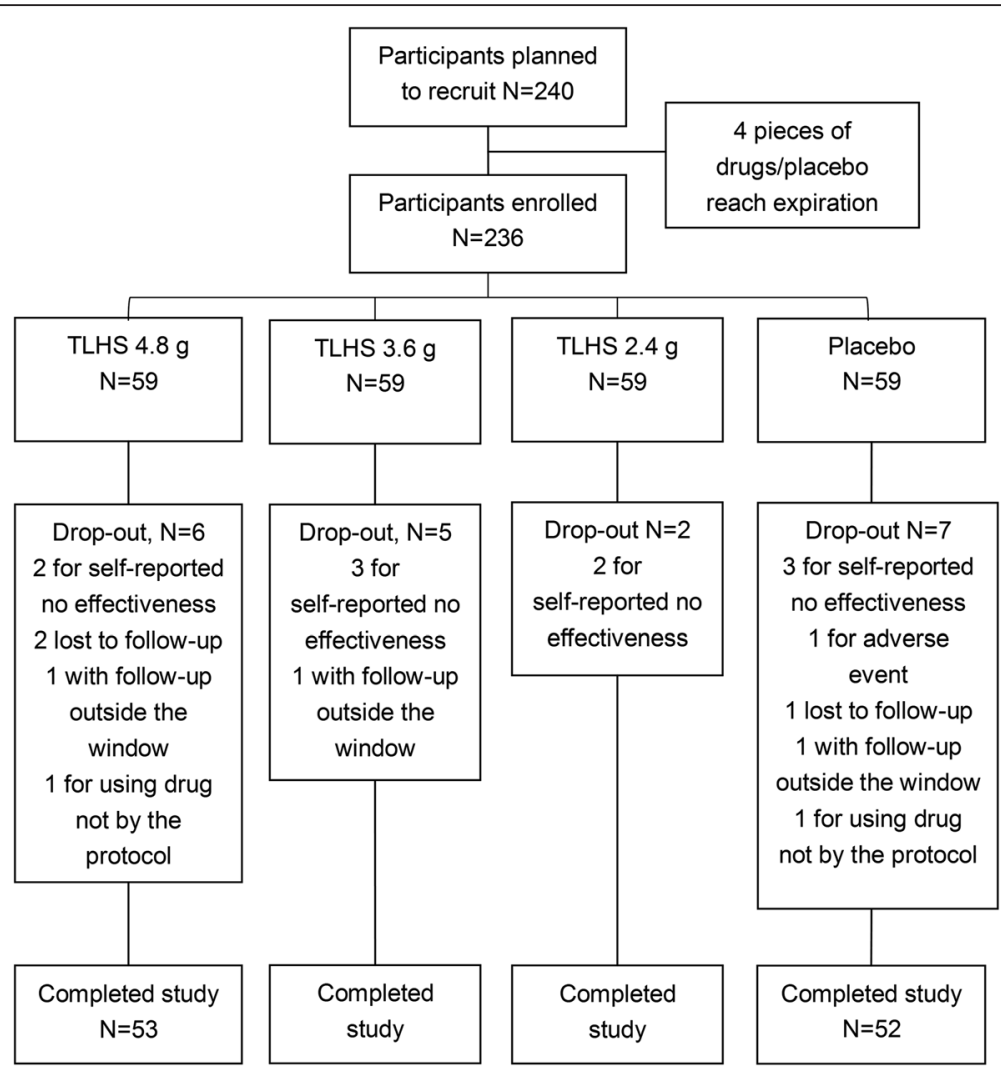

Fig. 1 Flowchart of the participants

Table 1 Baseline characteristics of the participants

\begin{tabular}{|c|c|c|c|c|}
\hline Characteristic & TLHS $4.8 \mathrm{~g}$ & TLHS $3.6 \mathrm{~g}$ & TLHS $2.4 \mathrm{~g}$ & Placebo \\
\hline Age, years, mean $\pm S D$ & $47.54 \pm 11.14$ & $50.76 \pm 9.69$ & $50.59 \pm 10.61$ & $50.12 \pm 10.45$ \\
\hline Gender, $n, M: F$ & $12: 47$ & $11: 48$ & $8: 51$ & $7: 52$ \\
\hline Height, $\mathrm{cm}$, mean $\pm \mathrm{SD}$ & $162.88 \pm 6.49$ & $162.08 \pm 6.16$ & $161.78 \pm 5.40$ & $162.37 \pm 5.60$ \\
\hline Weight, $\mathrm{kg}$, mean $\pm \mathrm{SD}$ & $61.83 \pm 9.87$ & $61.04 \pm 9.65$ & $60.14 \pm 9.04$ & $61.88 \pm 9.29$ \\
\hline Marital status, married : other & $53: 6$ & $56: 3$ & $55: 4$ & $56: 3$ \\
\hline Duration of disease, months & $3.00-180.00$ & $2.00-216.00$ & $5.00-183.00$ & $2.00-276.00$ \\
\hline Medication, use : no use & $20: 39$ & $28: 31$ & $32: 27$ & $28: 31$ \\
\hline Radiographic stage, I : || : || & $30: 25: 4$ & $23: 32: 4$ & $27: 22: 10^{*}$ & $33: 21: 5$ \\
\hline Joint functional stage, $1: \|$ : III & $13: 46: 0$ & $9: 47: 3$ & $9: 42: 8$ & $15: 40: 4$ \\
\hline Pain (VAS), $\mathrm{cm}$, mean $\pm \mathrm{SD}$ & $5.68 \pm 1.12$ & $5.68 \pm 1.27$ & $5.48 \pm 1.19$ & $5.52 \pm 1.13$ \\
\hline Tender joint counts, $n$, mean $\pm S D$ & $9.12 \pm 5.07$ & $8.47 \pm 5.06$ & $7.36 \pm 3.87$ & $9.24 \pm 5.33$ \\
\hline Swollen joint counts, $n$, mean \pm SD & $6.27 \pm 3.47$ & $5.83 \pm 3.34$ & $5.20 \pm 2.54$ & $6.19 \pm 3.57$ \\
\hline Morning stiffness, min, mean $\pm S D$ & $82.03 \pm 37.73$ & $81.36 \pm 37.61$ & $83.31 \pm 50.88$ & $90.25 \pm 71.24$ \\
\hline Grip strength, $\mathrm{mmHg}$, mean $\pm \mathrm{SD}$ & $36.11 \pm 25.84$ & $32.53 \pm 22.57$ & $35.07 \pm 24.00$ & $34.87 \pm 24.97$ \\
\hline Physician's assessments, score, mean \pm SD & $5.32 \pm 1.12$ & $5.34 \pm 1.33$ & $5.07 \pm 1.11$ & $5.30 \pm 1.17$ \\
\hline $\mathrm{HAQ}$, score, mean $\pm \mathrm{SD}$ & $16.68 \pm 11.87$ & $18.10 \pm 12.78$ & $15.39 \pm 11.09$ & $17.07 \pm 12.85$ \\
\hline $\mathrm{RF}, \mathrm{U} / \mathrm{ml}$, mean $\pm \mathrm{SD}$ & $163.69 \pm 258.04$ & $74.72 \pm 110.88$ & $99.19 \pm 149.96$ & $79.04 \pm 131.09$ \\
\hline $\mathrm{CRP}, \mathrm{mg} / \mathrm{dl}$, mean $\pm \mathrm{SD}$ & $7.38 \pm 15.13$ & $11.11 \pm 30.01$ & $6.83 \pm 12.42$ & $7.49 \pm 16.67$ \\
\hline $\mathrm{ESR}, \mathrm{mm} / \mathrm{h}$, mean $\pm \mathrm{SD}$ & $22.39 \pm 19.73$ & $27.21 \pm 25.14$ & $27.93 \pm 25.00$ & $26.39 \pm 23.04$ \\
\hline
\end{tabular}

*P $<0.05$ versus the placebo group

VAS visual analog scale, $H A Q$ Health Assessment Questionnaire, $R F$ rheumatoid factor, CRP C-reactive protein, ESR erythrocyte sedimentation rate 
Table 2 Adverse events and adverse drug reactions

\begin{tabular}{lllll}
\hline Event/reaction & TLHS $4.8 \mathrm{~g}$ & TLHS $3.6 \mathrm{~g}$ & TLHS $2.4 \mathrm{~g}$ & Placebo \\
\hline Adverse events, $n(\%)$ & $3(5.1)$ & $2(3.4)$ & $3(5.1)$ & $3(5.1)$ \\
$\begin{array}{l}\text { Adverse drug reactions, } \\
n(\%)\end{array}$ & $2(3.4)$ & $1(1.7)$ & $2(3.4)$ & $1(1.7)$ \\
\hline
\end{tabular}

According to the pain status and for all patients, including the patients in the placebo group, $25 \mathrm{mg}$ of diclofenac sodium enteric-coated tablets (Voltaren, Beijing Novartis Pharmaceutical Co., Ltd., Beijing, China) bid or tid, and/or $20 \mathrm{mg}$ of leflunomide (Fujian Huitian Biological Pharmaceutical Co., Ltd., Fuzhou City, China) were allowed, if necessary, after the second week of treatment, according to the treating physician's judgment. The treating physician was blind to the study treatment.

\section{Endpoints}

The primary efficacy endpoint was the improvement in ACR20 $[18,19]$. Secondary endpoints were rheumatoid factor (RF) levels, C-reactive protein (CRP) levels, erythrocyte sedimentation rate (ESR), ACR50, ACR70, and safety.

\section{Efficacy and safety}

Safety was monitored until the last administration of TLHS or placebo. Frequency and severity of adverse effects and adverse drug reactions were observed at each visit. Clinical and laboratory tests such as tender and swollen joint counts based on Disease Activity Score 28 (DAS28) [20], morning stiffness, average grip strength of two hands, 100-mm visual analog scale (VAS), and Health Assessment Questionnaire (HAQ)-disability index, were assessed by the investigators at screening, baseline, and at weeks 2, 4, and 8. Standard hematological and biochemical tests and urine analysis were also performed at each study site.

\section{Statistical analysis}

No data about TLHS was available in the literature on which to base the power analysis. However, based on data about Wu-wei-gan-lu, we estimated that the effective rate should be about $50 \%$ in the control group and about 75 $80 \%$ in the 4.8 g group. By setting the power at $80 \%$, $\alpha$ at 0.05 , and the same sample size in each group, the minimal sample size for each group was between 39 and 59. By considering the drop-out rate, limitation of funds, and probable errors in estimating the effective rate, we decided to include 60 patients in each group.

The efficacy analysis was performed using the intentto-treat principle, which includes all randomized participants who underwent at least one post-treatment evaluation. The last observation carried forward method was used to substitute for missing data. The safety analysis was performed on all participants who received at least one dose of study medication.

Continuous data are presented as mean \pm standard deviation (SD) and were analyzed using one-way analysis of variance (ANOVA) and Tukey's post hoc test. Categorical data are presented as frequencies and were analyzed using the chi-square test or Fisher's exact test, as appropriate. Statistical testing, unless otherwise stated, was two-sided and used a $5 \%$ significance threshold. Data were analyzed with SAS 9.2 (SAS Institute, Cary, NC, USA).

\section{Results}

\section{Characteristics of the participants}

A total of 240 participants from five centers were screened between May 2009 and June 2011 and provided a written informed consent (Fig. 1); 216, 236, and 236 participants were included in the per-protocol set (PPS), the full analysis set (FAS), and the safety set (SS), respectively. Four patients could not be randomized because four sets of drugs/placebo (one in each group) had reached the expiration date before recruiting a subject. The decision was then taken to stop the study because the scientific

Table 3 Effective rate according to the ACR20, ACR50, and ACR70

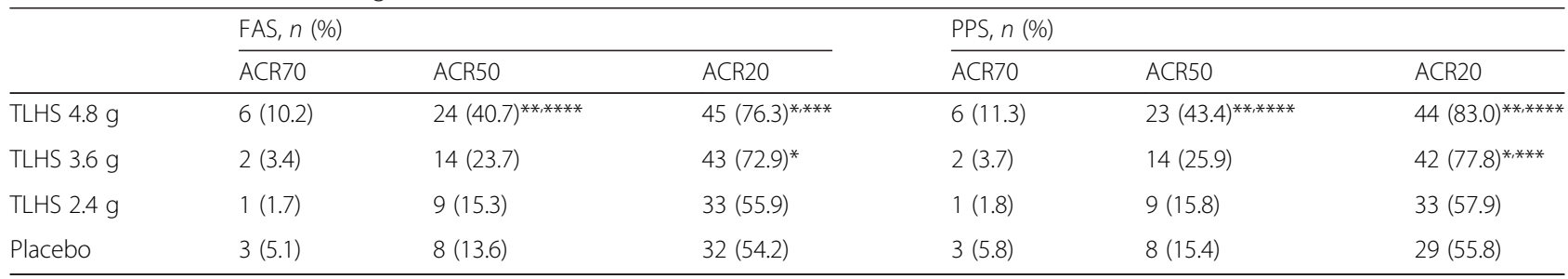

$A C R$ American College of Rheumatology response criteria, FAS full analysis set, PPS per protocol set

${ }^{*} P<0.05$ versus the placebo group

${ }^{*} P<0.01$ versus the placebo group

*** $P<0.05$ versus the $2.4 \mathrm{~g}$ group

**** $P<0.01$ versus the $2.4 \mathrm{~g}$ group 
Table 4 Condition of the participants at end of the trial

\begin{tabular}{|c|c|c|c|c|c|c|c|c|}
\hline \multirow[t]{2}{*}{ Indications } & \multicolumn{4}{|l|}{ FAS } & \multicolumn{4}{|l|}{ PPS } \\
\hline & TLHS $4.8 \mathrm{~g}$ & TLHS $3.6 \mathrm{~g}$ & TLHS $2.4 \mathrm{~g}$ & Placebo & TLHS $4.8 \mathrm{~g}$ & TLHS $3.6 \mathrm{~g}$ & TLHS $2.4 \mathrm{~g}$ & Placebo \\
\hline Pain (VAS), $\mathrm{cm}$, mean \pm SD & $2.62 \pm 1.37^{* *}$ & $3.31 \pm 1.30^{* *}$ & $3.64 \pm 1.29$ & $3.99 \pm 1.42$ & $2.48 \pm 1.30^{* *}$ & $3.24 \pm 1.32^{*}$ & $3.62 \pm 1.30$ & $3.89 \pm 1.45$ \\
\hline Pain changes, $\mathrm{cm}$, mean $\pm \mathrm{SD}$ & $-3.06 \pm 1.95^{* *}$ & $-2.37 \pm 1.31^{* *}$ & $-1.85 \pm 1.51$ & $-1.53 \pm 1.70$ & $-3.31 \pm 1.87^{* *}$ & $-2.44 \pm 1.25^{*}$ & $-1.88 \pm 1.51$ & $-1.63 \pm 1.74$ \\
\hline Tender joint counts, $n$, mean $\pm S D$ & $3.88 \pm 3.73^{* *}$ & $4.80 \pm 3.24$ & $4.24 \pm 2.54^{*}$ & $5.63 \pm 4.10$ & $3.96 \pm 3.89$ & $4.80 \pm 3.38$ & $4.19 \pm 2.53$ & $5.67 \pm 4.32$ \\
\hline Tender joint changes, $n$, mean \pm SD & $-5.24 \pm 3.54^{* *}$ & $-3.68 \pm 3.19$ & $-3.12 \pm 2.88$ & $-3.61 \pm 3.51$ & $-5.66 \pm 3.46$ & $-3.81 \pm 3.24$ & $-3.21 \pm 2.88$ & $-3.96 \pm 3.54$ \\
\hline Swollen joint counts, $n$, mean \pm SD & $2.24 \pm 2.09^{* *}$ & $2.68 \pm 1.82$ & $3.03 \pm 1.88$ & $3.41 \pm 2.49$ & $2.30 \pm 2.13$ & $2.56 \pm 1.77$ & $3.02 \pm 1.90$ & $3.31 \pm 2.47$ \\
\hline Swollen joint changes, $n$, mean $\pm S D$ & $-4.03 \pm 3.58^{*}$ & $-3.15 \pm 2.48$ & $-2.17 \pm 2.43$ & $-2.78 \pm 2.96$ & $-4.38 \pm 3.61^{*}$ & $-3.31 \pm 2.43$ & $-2.30 \pm 2.37$ & $-3.04 \pm 3.01$ \\
\hline Morning stiffness, min, mean $\pm S D$ & $36.53 \pm 24.69^{* *}$ & $46.10 \pm 26.57$ & $50.14 \pm 36.08$ & $54.93 \pm 41.78$ & $35.00 \pm 23.27^{* *}$ & $45.56 \pm 27.29$ & $50.14 \pm 36.67$ & $55.21 \pm 44.38$ \\
\hline Morning stiffness changes, min, mean $\pm S D$ & $-45.51 \pm 34.71$ & $-35.25 \pm 21.40$ & $-33.17 \pm 28.42$ & $-35.32 \pm 42.23$ & $-50.09 \pm 33.56$ & $-38.15 \pm 19.96$ & $-33.81 \pm 28.70$ & $-39.12 \pm 43.33$ \\
\hline Grip strength, mmHg, mean $\pm \mathrm{SD}$ & $47.68 \pm 28.59$ & $38.80 \pm 24.49$ & $40.58 \pm 26.27$ & $38.84 \pm 25.12$ & $47.14 \pm 29.13$ & $36.00 \pm 23.63$ & $39.63 \pm 26.22$ & $36.99 \pm 25.07$ \\
\hline Grip strength changes, $\mathrm{mmHg}$, mean $\pm \mathrm{SD}$ & $11.57 \pm 21.19^{* *}$ & $6.27 \pm 7.07$ & $5.51 \pm 10.01$ & $3.97 \pm 8.53$ & $12.32 \pm 22.21^{* *}$ & $6.76 \pm 6.98$ & $5.70 \pm 10.13$ & $3.93 \pm 8.75$ \\
\hline Physician's assessments, score, mean \pm SD & $2.65 \pm 1.40^{* *}$ & $3.32 \pm 1.37$ & $3.62 \pm 1.34$ & $3.79 \pm 1.42$ & $2.52 \pm 1.34^{* *}$ & $3.24 \pm 1.37$ & $3.62 \pm 1.36$ & $3.68 \pm 1.45$ \\
\hline Physician's assessments changes, score, mean \pm SD & $-2.67 \pm 1.91^{* *}$ & $-2.02 \pm 1.36$ & $-1.45 \pm 1.48$ & $-1.51 \pm 1.65$ & $-2.92 \pm 1.84^{* *}$ & $-2.07 \pm 1.30$ & $-1.46 \pm 1.49$ & $-1.60 \pm 1.71$ \\
\hline $\mathrm{HAQ}$, score, mean $\pm \mathrm{SD}$ & $10.31 \pm 9.91$ & $12.69 \pm 11.05$ & $11.36 \pm 9.64$ & $12.92 \pm 11.35$ & $11.19 \pm 10.06$ & $12.89 \pm 11.25$ & $11.65 \pm 9.67$ & $13.60 \pm 11.64$ \\
\hline HAQ changes, score, mean \pm SD & $-6.37 \pm 8.60$ & $-5.41 \pm 6.98$ & $-4.03 \pm 5.60$ & $-4.15 \pm 7.90$ & $-6.89 \pm 8.89$ & $-5.83 \pm 7.13$ & $-4.18 \pm 5.64$ & $-4.31 \pm 8.13$ \\
\hline $\mathrm{RF}, \mathrm{U} / \mathrm{ml}$, mean $\pm \mathrm{SD}$ & $122.61 \pm 194.75$ & $60.55 \pm 99.04$ & $90.35 \pm 160.68$ & $64.88 \pm 102.79$ & $129.88 \pm 203.21$ & $63.42 \pm 104.21$ & $93.28 \pm 165.28$ & $67.93 \pm 109.24$ \\
\hline $\mathrm{CRP}, \mathrm{mg} / \mathrm{dl}$, median $\pm \mathrm{IQR}$ & $12.63 \pm 44.50$ & $5.68 \pm 10.62$ & $5.48 \pm 8.48$ & $6.81 \pm 15.15$ & $14.05 \pm 47.27$ & $5.75 \pm 11.03$ & $5.78 \pm 8.70$ & $7.25 \pm 16.20$ \\
\hline $\mathrm{ESR}, \mathrm{mm} / \mathrm{h}$, mean $\pm \mathrm{SD}$ & $21.24 \pm 21.91$ & $21.32 \pm 24.15$ & $22.95 \pm 20.24$ & $23.25 \pm 19.66$ & $20.69 \pm 21.89$ & $21.39 \pm 25.25$ & $23.75 \pm 20.42$ & $24.26 \pm 20.56$ \\
\hline Joint functional stage, I : || : ||| & $22: 37: 0$ & 14:44:1 & $16: 40: 3$ & $20: 37: 2$ & 21:32:0 & 14:39:1 & $16: 38: 3$ & 19:32:1 \\
\hline Voltaren use : no use & $3: 56^{* *}$ & $10: 47^{* *}$ & $20: 38^{* *}$ & 23:33 & $3: 50^{* *}$ & $9: 45^{* *}$ & $20: 37$ & $21: 31$ \\
\hline Frequency of Voltaren use, mean \pm SD & $7.40 \pm 8.08^{* *}$ & $17.80 \pm 13.02^{* *}$ & $22.03 \pm 17.11^{* *}$ & $39.32 \pm 20.13$ & $7.40 \pm 8.08^{* *}$ & $17.63 \pm 13.35^{* *}$ & $22.03 \pm 17.11^{* *}$ & $39.29 \pm 20.34$ \\
\hline Leflunomide use : no use & 14:45 & $22: 35$ & $22: 36$ & $24: 32$ & 41:39 & $20: 34$ & $22: 35$ & $22: 30$ \\
\hline
\end{tabular}

FAS full analysis set, PPS per protocol set, VAS visual analog scale, HAQ Health Assessment Questionnaire, RF rheumatoid factor, CRP C-reactive protein, ESR erythrocyte sedimentation rate

${ }^{*} P<0.05$ and ${ }^{*} P<0.01$ versus the placebo group. There were 226 patients in these four groups. Ten patients dropped out before the first follow-up at 2 weeks. The other dropouts were included in this table using

the last follow-up data as the final data 
committee wanted to use the same batch of drugs for all patients. Twenty participants dropped out (Fig. 1).

Except for the radiographic stage $(P=0.031)$, there was no clinically significant difference between the groups at baseline (all $P>0.10)$ (Table 1).

\section{Safety}

Treatment tolerance was good. In the TLHS 4.8 g group, one case of insomnia and one case of gastroenteric reaction were reported. In the $3.6 \mathrm{~g}$ group, there was one case of arrhythmia. In the $2.4 \mathrm{~g}$ group, one case of upset stomach and one case of minor hepatic lesion were reported. A case of hepatic dysfunction was reported in the placebo group. There was no significant difference in the incidences of adverse events $(P=0.963)$ or adverse drug reactions $(P=0.878)$ between the groups (Table 2$)$. No serious adverse event occurred in the trial.

\section{Effective rate of the ACR improvement}

After 8 weeks, the improvement rates of the ACR20 in the TLHS $4.8 \mathrm{~g}$ and $3.6 \mathrm{~g}$ groups were significantly higher than in the placebo group $(P=0.012$ and $P=$ 0.035 in FAS, respectively; $P=0.002$ and $P=0.016$ in PPS, respectively). The improvement rate of the ACR20 in the 4.8 g group was higher than in the TLHS $2.4 \mathrm{~g}$ group ( $P=0.020$ in FAS; $P=0.004$ in PPS). The improvement rate of the ACR50 in the TLHS $4.8 \mathrm{~g}$ group was significantly higher compared with the placebo group $(P=$ 0.001 in FAS; $P=0.002$ in PPS) and the TLHS 2.4 g group $(P=0.002$ in FAS; $P=0.001$ in PPS) (Table 3$)$.

\section{Secondary outcomes}

The symptoms of the participants after treatments were significantly relieved. There were significant differences in the symptoms of RA between the TLHS groups and the placebo group after 8 weeks of treatments (Table 4).

\section{Discussion}

TLHS is a new formulation of the traditional Tibetan medicine Wu-wei-gan-lu decoction that has been used for the treatment of RA for hundreds of years in China. This study aimed to evaluate the efficacy and safety of TLHS in patients with RA. Results showed that after 8 weeks, ACR20 improvement in the TLHS $4.8 \mathrm{~g}$ and $3.6 \mathrm{~g}$ groups was significantly higher than in the placebo group. ACR50 improvement in the TLHS 4.8 g group was significantly higher compared with the placebo group. There was no difference in adverse events between the groups. TLHS improved the symptoms of patients with RA according to the ACR20. Moreover, TLHS was safe.

The quality of TLHS in China is controlled by HPLC analysis [21, 22]. In previous pharmacodynamics studies, TLHS revealed obvious inhibitory effects on swollen feet induced by albumen, formaldehyde, or adjuvant injections, and on edema of the ears of mice induced by xylene $[23,24]$. In addition, studies have shown that TLHS reduced the writhing responses to acetic acid injection and tail flick against thermal stimulation [10, 25-29]. It can also increase the coagulation time and regulate the phagocytic immunity of macrophages in mice. Toxicology studies suggested that after giving high, moderate, or low doses of TLHS for 6 months, the hematologic, biochemical, and histopathological indexes were normal [10, 25-29]. Furthermore, experimental data showed that the maximum tolerable dose of TLHS was as high as $75.6 \mathrm{~g} / \mathrm{kg}$, which is equivalent to 300 times the clinical dose for adults. Previous clinical trials showed that the basic formula of TLHS, Wuwei-gan-lu decoction, has immunoregulatory and antiarthritic effects for treating RA [14, 15].

The present study suggests that TLHS may be used to treat the symptoms of RA and to improve the quality of life, and that it is safe. On one hand, it demonstrated a good mid-term safety profile and good treatment compliance, in spite of some mild and transient adverse events or reactions including insomnia, gastroenteric reactions, and arrhythmias. On the other hand, the symptoms such as pain, tenderness, swelling, and morning stiffness were improved by TLHS. The patients' grip strength and their conditions assessed by the physician could also be improved. Finally, fewer painkillers were needed by the patients. Nevertheless, it must be stressed that these results were obtained in a Chinese population and that traditional Chinese medicine is seldom used in Western countries.

The present study is not without limitations. Even if it was a multicenter trial, the sample size was relatively small. Second, only a limited panel of inflammatory markers was assessed. Further study is necessary to determine the comprehensive mechanisms of TLHS on inflammation. Larger studies are necessary to adequately demonstrate the effects of TLHS on RA. Other chronic inflammatory conditions might also benefit from TLHS.

\section{Conclusions}

The results of the present study showed that TLHS is safe and effective in improving the primary effective values and reducing the secondary symptoms in patients with RA.

\section{Additional files}

Additional file 1: CONSORT 2010 Checklist. (DOC $217 \mathrm{~kb}$ )

Additional file 2: CONSORT flow diagram. (PDF $483 \mathrm{~kb}$ )

\section{Abbreviations}

ACR, American College of Rheumatology; DMARD, disease-modifying antirheumatic drug; FAS, full analysis set; HAQ, Health Assessment 
Questionnaire; NSAID, Nonsteroidal anti-inflammatory drug; PPS, per-protocol set; RA, rheumatoid arthritis; RF, rheumatoid factor; SS, safety set; TLHS, Tong Luo Hua Shi; VAS, visual analog scale

\section{Acknowledgements}

The authors thank the participants who participated in the trial. They are also grateful to the staff of the participating centers.

\section{Funding}

This study was funded by the National Natural Science Foundation of China (No. 81273709 and No. 81473635) and the Pioneering Program of the First Teaching Hospital of Tianjin University of Traditional Chinese Medicine (No. 201309).

\section{Authors' contributions}

WL and YHW contributed to conception and design; WL, YHW, SYH, CLZ, MLG, DWL, HYW, MZC, YJS, BZXY, QSZ, HY, XBQ, and GL contributed to acquisition of data, or analysis and interpretation of data; WL and YHW have been involved in drafting the manuscript or revising it critically for important intellectual content; all authors have given final approval of the version to be published.

\section{Competing interests}

The authors declare that they have no competing interests.

\section{Author details}

'First Teaching Hospital of Tianjin University of Traditional Chinese Medicine, An-shan-xi Road \#314, Nankai District, Tianjin 300193, China. ${ }^{2}$ Affiliated Hospital of Liaoning University of Traditional Chinese Medicine, Shenyang, China. ${ }^{3}$ Traditional Chinese Medicine Hospital of Xinjiang Uygur Autonomous Region, Urumqi, China. ${ }^{4}$ Hubei Hospital of Traditional Chinese Medicine, Wuhan, China. ${ }^{5}$ Tibetan Medicine Hospital of Qinghai Province, Xi'ning, China. ${ }^{6}$ Center for Drug Clinical Research, Shanghai University of Traditional Chinese Medicine, Shanghai, China. 'Lanzhou Heshengtang Pharmaceutical Co. Ltd., Lanzhou, China. ${ }^{8}$ Beijing Highthink Pharmaceutical Service Technology Co. Ltd., Beijing, China.

Received: 21 October 2015 Accepted: 11 June 2016

Published online: 27 July 2016

\section{References}

1. Klarenbeek NB, Kerstens PJ, Huizinga TW, Dijkmans BA, Allaart CF. Recent advances in the management of rheumatoid arthritis. BMJ. 2010;341:C6942

2. Rindfleisch JA, Muller D. Diagnosis and management of rheumatoid arthritis. Am Fam Physician. 2005;72:1037-47.

3. Lane SK, Gravel Jr JW. Clinical utility of common serum rheumatologic tests. Am Fam Physician. 2002;65:1073-80.

4. Cross M, Smith E, Hoy D, Carmona L, Wolfe F, Vos T, et al. The global burden of rheumatoid arthritis: estimates from the global burden of disease 2010 study. Ann Rheum Dis. 2014;73:1316-22.

5. Sugiyama D, Nishimura K, Tamaki K, Tsuji G, Nakazawa T, Morinobu A, et al. Impact of smoking as a risk factor for developing rheumatoid arthritis: a meta-analysis of observational studies. Ann Rheum Dis. 2010;69:70-81.

6. Turesson C, Matteson EL. Genetics of rheumatoid arthritis. Mayo Clin Proc. 2006;81:94-101.

7. Nielsen SF, Bojesen SE, Schnohr P, Nordestgaard BG. Elevated rheumatoid factor and long term risk of rheumatoid arthritis: a prospective cohort study. BMJ. 2012;345:e5244

8. Heliovaara M, Aho K, Knekt P, Impivaara O, Reunanen A, Aromaa A. Coffee consumption, rheumatoid factor, and the risk of rheumatoid arthritis. Ann Rheum Dis. 2000:59:631-5.

9. Boscarino JA, Forsberg CW, Goldberg J. A twin study of the association between PTSD symptoms and rheumatoid arthritis. Psychosom Med. 2010;72:481-6.

10. Jia J. The prescription of "Five-tastes nectar" (bdud-rtsi Inga-lums) and Tibetan herbal bath. China Tibetology. 2007;79:157-61.

11. Huang F. On the academic connotation and development of Tibetan medicinal balneum. China Tibetology. 2002;58:26-32.

12. Huang F. Study on Origin of bdud rtsi rnam pa Inga in bathing treatment of Tibetan medicine. China Tibetology. 2002;58:129-38.

13. Liang HD, Wang JB, Pan L. Clinical trial of Wuweiganlu Yaoyu Keli for acute gouty arthritis. Chinese J Gen Pract. 2011;9:1863-1865.

14. Liu W, Gao CH, Xue B. Clinical research of effects of Wuweiganlu particles dipping on rheumatoid arthritis. Chinese J Gen Pract. 2012;10:855-856.
15. Bao YH, Wu LJ, Bao WL. The progress in Mongolian Medicine Treatment of Rheumatoid Arthritis. J Medicine Pharm Chinese Minorities. 2013;19:59-60.

16. Schulz KF, Altman DG, Moher D, Group C. CONSORT 2010 statement: updated guidelines for reporting parallel group randomised trials. BMJ. 2010;340:c332.

17. Hochberg MC, Chang RW, Dwosh I, Lindsey S, Pincus T, Wolfe F. The American College of Rheumatology 1991 revised criteria for the classification of global functional status in rheumatoid arthritis. Arthritis Rheum. 1992:35:498-502.

18. Felson DT, Anderson J, Boers M, Bombardier C, Furst D, Goldsmith C, et al. American College of Rheumatology. Preliminary definition of improvement in rheumatoid arthritis. Arthritis Rheum. 1995;38:727-35.

19. Felson DT, Anderson JJ, Lange ML, Wells G, LaValley MP. Should improvement in rheumatoid arthritis clinical trials be defined as fifty percent or seventy percent improvement in core set measures, rather than twenty percent? Arthritis Rheum. 1998:41:1564-70.

20. Fransen J, Creemers MC, Van Riel PL. Remission in rheumatoid arthritis: agreement of the disease activity score (DAS28) with the ARA preliminary remission criteria. Rheumatology (Oxford). 2004;43:1252-5.

21. Chinese Pharmacopoeia Commission. Pharmacopoeia of the People's Republic of China. Volume I. Chemical Industry Press: Beijing; 2010.

22. Zhang $X$. The quality standard for the Tong Luo Hua Shi Capsule. Chinese J Exper Traditional Medical Formulae. 2010;16:75-6.

23. Jiang J, Xu Q. Immunomodulatory activity of the aqueous extract from rhizome of Smilax glabra in the later phase of adjuvant-induced arthritis in rats. J Ethnopharmacol. 2003:85:53-9.

24. Xu Q, Wang $R, X u L$, Jiang J. Effects of Rhizoma smilacis glabrae, on cellular and humoral immune responses. Chinese J Immunology. 1993;9:39-42.

25. Li G, Guo J, Li P. The anti-inflammatory effect of Wu Wei Gan Lu medicated foam. Chinese Traditional Pat Medicine. 2001:23:224-5.

26. Zhuoma L. Study on the Wu Wei Gan Lu medicated bath of Tibetan Medicine. J Medicine and Pharmacy of Chinese Minorities. 2013;19:28-31.

27. Pbucairan. Tibetan medicine herbal bath treatment of 30 cases of rheumatic and rhumatoid arthritis. J Medicine and Pharm Chinese Minorities. 2008;14:17-8.

28. Ma C. Wu Wei Gan Lu medicated bath of Tibetan medicine and its clinical study. The 1st Discussion Forum of Chinese National Medicine. Beijing: 1996

29. Li X. Tibetan medicine herbal bath treatment of 223 cases of rheumatic and rheumatoid arthritis. J Medicine and Pharm Chinese Minorities. 1997:3:15-6.

\section{Submit your next manuscript to BioMed Central and we will help you at every step:}

- We accept pre-submission inquiries

- Our selector tool helps you to find the most relevant journal

- We provide round the clock customer support

- Convenient online submission

- Thorough peer review

- Inclusion in PubMed and all major indexing services

- Maximum visibility for your research

Submit your manuscript at www.biomedcentral.com/submit
) Biomed Central 